\title{
¿Eco-innovación, una evolución de la innovación? Análisis empírico en la industria cerámica española
}

\author{
M. SEGARRA-OÑA'1', A. PEIRÓ-SIGNES' ${ }^{1}$, L. MIRET-PASTOR², J. ALBORS-GARRIGÓS ${ }^{1}$ \\ 'Departamento de Organización de Empresas, Escuela Técnica Superior de Ingenieros Industriales. Universidad Politécnica de Valencia. \\ ${ }^{2}$ Departamento de Economía y Ciencias Sociales, Escuela Politécnica Superior de Gandía. Universidad Politécnica de Valencia
}

\begin{abstract}
La innovación y el desarrollo sostenible se consideran motores económicos cruciales en la fijación de la posición competitiva de las empresas. La eco-innovación, entendida como relación sinérgica entre ambos conceptos, debe ser un elemento a considerar en el diseño de la estrategia de las empresas. El objetivo de este trabajo es analizar cuáles son las variables que determinan que las empresas innovadoras vayan más allá y consideren la mejora del impacto ambiental como un objetivo al desarrollar actividades innovadoras. En este trabajo se analizan las empresas pertenecientes al sector cerámico español. Los datos provienen de la base de datos PITEC. El documento identifica los factores moderadores que influyen en el comportamiento eco- innovador de las empresas.
\end{abstract}

Palabras clave: Eco-innovación, Medio ambiente, Sector cerámico.

Eco-innovation an evolution of innovation? Empirical analysis at the Spanish tile ceramic industry.

Innovation and sustainable development are considered to be economic drivers and crucial in fixing competitive position of companies. Eco-innovation, known as a synergic relation among both concepts must be an element to consider when designing the company's strategy. The objective of this paper is to analyze which are the variables that determine that innovative companies go beyond and consider the improvement of their environmental impact as an output when developing innovating activities. This research considers firms belonging to the Spanish tile industry. Data has beeen provided by PITEC Database. The paper identifies the moderating factors that influence the eco-innovative behavior of firms.

Keywords: Eco-innovation, environment, ceramic industry.

\section{INTRODUCCIÓN}

La relación positiva entre la innovación y la competitividad se ha estudiado de manera amplia $(1,2,3,4)$. La innovación genera beneficios asociados a los costes, la productividad o el acceso a nuevos mercados $(5,6$,$) y representa un aspecto clave$ de la competitividad $(7,8)$ y el crecimiento (9). Estos impactos positivos explican el interés de la literatura académica por identificar los factores que contribuyen a la mejora y al fomento de la innovación $(10,11)$.

La innovación y la sostenibilidad son dos conceptos que por separado impactan en la posición competitiva de las empresas (12) y que, juntos, actúan de forma sinérgica (13) generando, por un lado, nuevos mercados para los productos ambientalmente benignos (14) y un nuevo campo de estudio académico, la eco-innovación $(15,16,17,18)$. La intersección entre la empresa y el medio ambiente está transformando los mercados existentes, creando nuevos y, cada vez más, incluyendo los principios de sostenibilidad en las estrategias de negocio empresariales (19).

Las empresas han identificado los beneficios de la conducta innovadora $(7,20)$ pero, ¿qué se necesita para pasar de conductas innovadoras a un comportamiento eco-innovador?, ¿Qué aspectos determinan que las empresas que crean valor a través de la innovación tengan un enfoque proactivo hacia el medio ambiente?, más específicamente, ¿Cuáles son los factores moderadores que ayudan a las empresas innovadoras a convertirse en eco-innovadoras? El objetivo de este trabajo es identificar aquellos aspectos que determinan si una empresa, como resultado de su actividad innovadora, reduce al mínimo su impacto ambiental, pasando de ser innovadora a ser ecoinnovadora. Para resolver las cuestiones de investigación planteadas, se han analizado los datos recogidos en el Panel de Innovación Tecnológica, PITEC, edición 2007, una base de datos que recoge las actividades de innovación tecnológica de las empresas españolas. Así, se determinará si es posible identificar factores moderadores (21), que influyan en esta relación.

Este documento está estructurado de la siguiente manera. En primer lugar se define el concepto de eco-innovación y se plantea el objetivo de la investigación. A continuación se analiza el estado del arte del concepto eco-innovación, los indicadores utilizados con anterioridad para medirla, así como las barreras y los facilitadores previamente identificados a su implantación. Posteriormente se plantean las hipótesis y se describe la metodología de investigación y los resultados del trabajo de campo y, por último, tras el análisis estadístico y la discusión de los resultados, se presentan las conclusiones. 


\section{EL CONCEPTO DE LA ECO-INNOVACIÓN EN EL CONTEXTO INSTITUCIONAL Y ACADÉMICO.}

La Unión Europea (UE) ha impulsado varios grupos de reflexión para analizar y promover la eco-innovación como el "Foro Europeo de Eco-innovación" o "Clusters y regiones para el fomento de la red europea eco-innovadora y eco-inversora", así como trabajos académicos centrados en la definición y medición de la eco-innovación $(22,23)$. Tanto el esfuerzo económico como el interés institucional justifican, en gran medida, el interés de la comunidad académica y empresarial. A nivel científico están empezando a aparecer trabajos ligados ya no sólo a la innovación, sino también a la eco--innovación, aunque aún existe una brecha importante en el estudio de las conexiones y las diferencias entre el rendimiento y el comportamiento entre las empresas innovadoras y las eco-innovadoras.

Varios autores $(20,24)$ han utilizado la base de datos PITEC para avanzar en la comprensión de la innovación en las empresas, así como en la relación entre las diferentes estrategias innovadoras, pero aún no hay trabajos que analicen el comportamiento eco-innovador de los sectores industriales españoles, por lo que en esta investigación nos planteamos analizar el comportamiento medioambiental de las empresas productoras de azulejos y baldosas cerámicas.

El papel de la gestión medioambiental en el proceso de creación de valor es fundamental $(25,26)$ ya que implica tomar decisiones en ámbitos indirectamente relacionados con el medioambiente, tales como compras, logística, diseño de producto, o actividad de I $+\mathrm{D}$, pero con un impacto relevante en una gestión ambiental global (27).

Aunque la investigación sobre la gestión ambiental es relativamente nueva, en la actualidad es un campo científico de gran interés tanto para las grandes empresas, como para las pequeñas y medianas $(28,29)$. Abbaspour et al $(30)$ definen las directrices para el establecimiento de sistemas de gestión verdes en complejos deportivos. Beveridge y Guy (31) analizan la relación entre los eco-emprendedores y la innovación sostenible. Jänicke (18) aborda la cuestión de la mejora sostenible continua, entendida como la difusión sistemática de eco-innovaciones y Lucas (32) establece un modelo integrado basado en la teoría de recursos y capacidades (RBV). Aunque los temas relacionados con la sostenibilidad y lo "eco" son cruciales tanto para los gobiernos, como para las industrias y las empresas, las características específicas que pueden contribuir a mejorar su desarrollo no se han estudiado a fondo todavía.

\subsection{Eco-innovación: un paso por delante de la innovación.}

Se considera eco-innovación cualquier innovación que reduce el daño al medio ambiente (33). Una definición social es la establecida por Huppes et al (17), al definirla como un "cambio en las actividades económicas que implique una mejora del rendimiento tanto en los aspectos sociales y económicos como en los ambientales" y, más explícitamente, Kemp y Pearson (22) la definen como "la producción, la asimilación o la explotación de un producto, proceso de producción, servicio o método de gestión o negocio que es nuevo para la organización y que implica, a lo largo de su ciclo de vida, una reducción de los riesgos ambientales, de la contaminación y de otros impactos negativos en el uso de los recursos (incluida la energía) en comparación con las alternativas correspondientes".

La eco-innovación ha recibido recientemente una considerable atención académica $(22,34,17)$, especialmente en lo que representa una ventaja competitiva que permite aumentar el valor añadido para los productores y los consumidores, reduciendo al mismo tiempo impactos ambientales, pero hasta ahora se ha estudiado desde el punto de vista anecdótico (35).

Varios autores han descrito la falta de indicadores, estadísticas y bases de datos específicas de eco-innovación a nivel europeo $(22,34)$. Posteriormente, los esfuerzos se han llevado a cabo para desarrollar sistemas de medición de la ecoinnovación, teniendo en cuenta todos los agentes pertenecientes al sistema; el sector financiero, la educación o las instituciones relacionadas con la gestión del conocimiento (36).

La literatura científica ha identificado varias características distintivas de las innovaciones medioambientales, como la doble generación de externalidades (14) considerando la mejora de la calidad del medio ambiente como un bien para la sociedad en su conjunto, aunque el coste sea soportado únicamente por el innovador, la dificultad para apropiarse de los beneficios por parte del innovador o su difusión en el mercado sin mayor promoción (37).

Por otro lado, el análisis de los gastos destinados a las actividades de innovación sostenibles se ha analizado por parte de varios autores. Jaffe y Palmer (38) realizaron un estudio empírico de la industria norteamericana manufacturera a través de un estudio de panel, encontrando una influencia positiva de los gastos ambientales en la investigación futura y el gasto en $\mathrm{I}+\mathrm{D}$, pero no en el número de solicitudes de patentes. Con el mismo tipo de estudio, utilizando datos de panel de 500 empresas norteamericanas, Wagner (39) concluyó que las actividades de innovación ambiental vienen determinadas por la intensidad de I $+\mathrm{D}$ total, ya que el análisis no encontró ninguna relación entre la gestión ambiental y la eco-innovación. Basándonos en el estudio de trabajos anteriores (40) consideramos que la eco-innovación se basa en actividades previas de innovación, y que puede ser considerada como un paso más allá de la innovación. Este es el tema de nuestro trabajo.

\subsection{La eco-innovación y la estructura interna de la empresa.}

En la literatura se han estudiado los cambios y las innovaciones organizativas en las empresas, relacionando algunas características de la tipología empresarial con el tipo de innovación llevada a cabo. Diversos estudios $(41,42)$ encontraron que las innovaciones incrementales se suelen adoptar por las empresas de mayor tamaño, mientras que las empresas más pequeñas tienden a introducir innovaciones radicales. Siguiendo la misma línea, Lazaric y Dennis (43), al analizar la introducción de las normas ambientales, se refirieron a la importancia de los procesos de mejora de la gestión para hacer frente a los cambios derivados de su aplicación y cómo establecer rutinas organizativas (44) se convierte en un aspecto clave para éxito de la innovación y la evolución de la organización (45). Desde nuestro punto de vista, estas cuestiones van a influir también en el desarrollo de la eco-innovación, al considerar el paso de la orientación innovadora a la eco-innovadora como un avance en la madurez organizativa (40). 
Reid y Männik (46) llevaron a cabo un estudio prospectivo sobre la eco-innovación y señalaron dos cuestiones clave como barreras internas: el acceso a los recursos humanos de nivel superior y las previsiones con respecto a la tecnología y a los mercados. Por otra parte, desde una perspectiva exógena, señalaron igualmente el acceso a los recursos humanos de nivel superior y la dificultad para acceder a la financiación adecuada, así como y el entorno jurídico y reglamentario a nivel europeo. Estos factores resultan aún más difíciles de superar en el caso de las PYMES $(47,48)$.

Por otra parte, en uno de los trabajos principales de Brunnermeier y Cohen (49) se identificaron los factores determinantes de la innovación medioambiental en la industria manufacturera de EE.UU., lo que abrió un nuevo campo de estudio e introdujo una nueva variable, al evidenciar que la innovación medioambiental es más probable que ocurra en los sectores competitivos a nivel internacional. Por último, los recursos y su acceso (47), el papel del tamaño de la empresa, la capacidad de integrar conocimientos, así como la disponibilidad de recursos humanos o de fondos económicos, también han sido subrayados (24) como cuestiones clave de la eco-innovación.

\section{HIPÓTESIS DE INVESTIGACIÓN.}

En las secciones anteriores hemos presentado la ecoinnovación como un nuevo paradigma que orienta el cambio hacia el desarrollo sostenible desde tres enfoques distintos: el social, el institucional y el tecnológico $(33,50)$. Tanto los cambios sociales como los cambios institucionales asociados a la innovación sostenible han sido ampliamente analizados (22; 30; 51), así como sus externalidades $(14,35,38)$, incluso las formas de aproximarse hacia la eco innovación por parte de las empresas $(40,52)$. Sin embargo, los aspectos asociados a los conductores y facilitadores de la eco-innovación en las empresas, así como a la afectación de las condiciones del entorno macroeconómico de la eco innovación en la empresa aún no se han analizado suficientemente. Este trabajo tiene como objetivo llenar el vacío de investigación detectado.

La primera hipótesis de investigación que nos planteamos está relacionada con los facilitadores de la eco-innovación, tales como las actividades de I+D $(38,39)$, al considerar que la estructura de innovación de una organización puede ser entendida como un paso más en la orientación hacia la eco-innovación, lo que nos conduce a plantear la primera hipótesis, H1: Cuanto más orientadas hacia la innovación están las empresas, más orientadas hacia la eco-innovación estarán.

Por otra parte, al examinar la estructura interna de la empresa y, teniendo en cuenta que ésta estará relacionada con su evolución $(44,45,53)$, así como con su tamaño, la pregunta de investigación que se nos plantea es sobre si esta variable puede suponer un obstáculo a la eco-innovación $(24,43,48$, 49). Sobre esta base, se postula la siguiente hipótesis, $H 2$ : $E l$ tamaño de la empresa determina la orientación hacia la innovación sostenible de la empresa.

Por último, y teniendo en cuenta lo que algunos autores han señalado sobre la relación entre la orientación ecoinnovadora de la empresa y su competitividad internacional $(1,35,49,50)$, la tercera hipótesis se refiere a la relación entre la empresa con orientación eco-innovadora y su vocación exportadora, proponiendo así la tercera hipótesis, H3: Cuanto

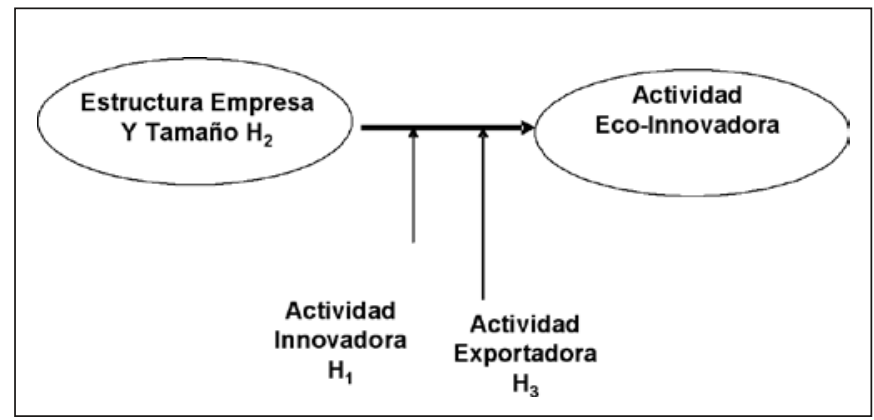

Figura 1. Modelización de los factores que afectan a la eco-innovación.

Fuente: Elaboración propia.

más orientadas a la exportación están las empresas, más sensibles son a los impactos de la eco-innovación. El modelo propuesto se presenta en la figura 1.

\section{METODOLOGÍA.}

España es el segundo productor europeo de baldosas cerámicas y el tercer exportador mundial, después de China e Italia. El sector español de la cerámica produjo en 2009 324,4 millones de $\mathrm{m}^{2}$ de baldosas cerámicas. Emplea a más de 17.500 personas directamente y cerca de 6.000 de manera indirecta (54) El sector cerámico español viene sufriendo fuertes descensos en su cifra de ventas y se enfrenta a importantes retos en la próxima década $(55,56)$. Esta situación de crisis necesita tener en cuenta las peculiaridades del sector, tanto a nivel de organización de su producción $(57,58)$, como de adopción de estrategias específicas a sus peculiaridades (59), así como replantearse las relaciones que establece con sus proveedores, distribuidores y clientes $(60,61)$ y encontrar nuevas opciones de generación de ventajas competitivas que le permitan aumentar el valor añadido de sus productos.

Los datos se han obtenido de la base de datos PITEC (Panel de Innovación Tecnológica), una herramienta de seguimiento estadístico de las actividades de innovación tecnológica de las empresas españolas. La base de datos ha sido construida por el INE (Instituto Nacional de Estadística) con el asesoramiento de expertos de Cotec (Fundación para la Innovación Tecnológica). El primer año del que se encuentran datos disponibles es el 2004, se actualiza anualmente e incluye una lista completa de empresas españolas, siendo el número total de variables analizadas de 255. Los datos provienen de una encuesta de tipo macroeconómico de carácter anónimo.

Del total de 8.870 empresas incluidas en la base de datos PITEC correspondiente al año 2007, se han seleccionado las pertenecientes a empresas productoras del sector cerámico, obteniendo una muestra de 61 empresas. La muestra se ha seleccionado atendiendo a la variable ACTI (actividad) que representa el código según la clasificación CNAE 2003. Para la selección de los casos se ha tomado aquellos pertenecientes a los códigos CNAE-2003 "AZULEJOS Y BALDOSA CERAMICA" (263) que no incluye los "PRODUCTOS MINERALES NO METALICOS (EXCEPTO AZULEJOS Y BALDOSA CERAMICA 26)". Para el análisis de los datos se ha utilizado una metodología de tipo cuantitativo en dos etapas. 
TABLA I. CLASIFICACIÓN DE ESTABLECIMIENTOS ESTUDIADOS SEGÚN EL TAMAÑO DE LA PLANTILLA.

Productores de azulejos y baldosas cerámicas

$\begin{array}{lcc}\text { TAMAÑO }<50 & 14 & 23 \% \\ \text { TAMAÑN } 50<\mathbf{x}<\mathbf{2 5 0} & 25 & 41 \% \\ \text { TAMAÑO }>\mathbf{2 5 0} & 22 & 36 \% \\ \text { TOTAL } & \mathbf{6 1} & \mathbf{1 0 0} \%\end{array}$

Fuente: Elaboración propia a partir de la base de datos PITEC (2007).

Es importante identificar las características de las empresas estudiadas ya que éstas afectan a sus prácticas de gestión ambiental. Todas las empresas analizadas se encuentran en España. En cuanto a la ubicación de las empresas, la base de datos PITEC 2007 no proporciona directamente datos referidos a la ubicación de la empresa, sin embargo el análisis de las variables PIDj y similares que relacionan el Personal total en $\mathrm{I}+\mathrm{D}$ por comunidades autónomas o el gasto en $\mathrm{I}+\mathrm{D}$ interna por comunidades autónomas permite deducir que al menos 28 de las empresas de la muestra está ubicadas en la Comunidad Valenciana.

La Tabla I muestra la tipología de empresas según el número de empleados en el sector estudiado y en el total del sector industrial español.

Dubé y Pare (62), afirman que en la investigación de tipo cuantitativo, métodos usuales y conocidos como el análisis de la varianza o las regresiones, ayudan a confirmar o refutar hipótesis. Así pues, en este estudio, en primer lugar se ha realizado un análisis factorial para reducir variables, lo que nos permite obtener grupos homogéneos de variables correlacionadas que condensa la información contenida en un grupo de variables originales en un conjunto más pequeño con una pérdida mínima de información. En la segunda fase del análisis, se realiza una regresión lineal que se ajusta a los datos, utilizando como variables los factores previamente identificados con el fin de analizar la relación existente entre la variable dependiente (denominada en la base de datos PITEC Efecto 8), que mide la orientación medioambiental de las empresas al innovar y las variables independientes.

\subsection{Selección de variables: descripción y adecuación a la investigación.}

Las variables incluidas en este estudio se seleccionaron de acuerdo a la teoría estudiada previamente. Las ventas netas (NS), representan el ingreso total por ventas en 2007, medido en euros, el tamaño por número de empleados, (SZ), representa el número de empleados a tiempo completo en la empresa durante el año 2007; la variable inversión en bienes, INVER, representa la inversión bruta, medida en euros, en bienes materiales en 2007; las variables MDONAC y MDOUE hacen referencia al enfoque de mercado nacional o comunitario de la empresa, indicando si el Enfoque comercial de la empresa es hacia el mercado de la Unión Europea a través de una variable binaria, 1 = Sí, 0 = No. El Número de patentes europeas, PATEPO y el Número Nacional de Patentes, PATOEPM, se miden como el número de patentes solicitadas en la Oficina Española de Patentes y Marcas (OEPM) y, a nivel europeo, las patentes solicitadas en la Oficina Europea de Patentes (EPO). Los indicadores de patentes en EE.UU. fueron descartados porque no tenía un valor significativo en la muestra estudiada. Las Innovaciones en el mercado, MERCADO, representa la cantidad total invertida en el lanzamiento de innovaciones de producto al mercado, medida en euros.

\subsection{Resultados y discusión.}

Para construir los indicadores, la información se ha extraido de la base de datos PITEC (2007) y fue procesada posteriormente. Como paso previo, se llevó a cabo un análisis factorial exploratorio sobre las ocho variables independientes, utilizando el método Varimax, tratando de entender la estructura de los factores y la medición de la calidad de los mismos. Los resultados obtenidos muestran tres factores que explican el $71,046 \%$ de la varianza con una significación positiva ( $\mathrm{p}<0.0001$ ). Estos factores se han denominado: tamaño, orientación al mercado exterior y actividad formal de innovación e inversión.

En la tabla II se muestran las cargas factoriales que representan los coeficientes de correlación entre las variables (filas) y los factores (columnas). Las cargas factoriales con valores superiores a 0,6 se consideran altos (63), permiten confirmar que las variables independientes identificadas a priori están representadas por un determinado factor e interpretar lo que representa el factor teniendo en cuenta aspectos teóricos. Se incluye el porcentaje de varianza explicada de cada factor y para facilitar la interpretación de los resultados se han surpimido las cargas factoriales bajas que son aquellas con influencia despreciable en cada factor.

Todos los análisis estadísticos se realizaron con SPSS para Windows, versión 17.0. Se utilizó el test de esfericidad de Barlett calculado con la opción Meyer-Olkin/estadístico Kaiser, para verificar la conveniencia del análisis. En el campo de estudio de las ciencias sociales, es válido aceptar una solución que explique más del 60\% de la varianza, (63). Las estimaciones de los factores, así como la evaluación del ajuste global se llevaron a cabo mediante el análisis de componentes principales, que resulta adecuado para resumir la información original de los factores para análisis futuros (63), como es el caso que nos ocupa.

Los resultados de la rotación Varimax refuerzan el patrón esperado: las ventas netas, el número de empleados y la inversión total, son características todas que hacen referencia al tamaño de la empresa y definen una nueva variable, Tamaño. Según la teoría, las grandes estructuras involucran a más empleados y generan mayor cantidad de ventas y, por otra parte, el tamaño de la empresa en el proceso de crecimiento está asociado a una mayor complejidad en su estructura organizativa y su compromiso con la innovación $(45,53,64)$. Todas las variables seleccionadas según el análisis de primeros componentes están correlacionadas positivamente reforzando la explicación en base a la teoría evolucionista de Nelson y Winter (44), así como a otros estudios más específicos, como el de Horbach (65), quién comprobó cómo las herramientas de gestión ambiental y en general los cambios organizativos, fomentan la innovación medioambiental.

Las variables que hacen referencia a la orientación e enfoque del mercado exterior, MDONAC (Enfoque hacia 
el mercado nacional) y, MDOUE (enfoque hacia el mercado europeo) se combinan conformando el segundo factor, que hemos llamado Apertura al mercado. Según la teoría, la innovación juega un papel crucial en el comportamiento de las exportaciones, comportándose como un factor moderador en las empresas orientadas a los mercados más abiertos $(66,67)$.

La Actividad formal de innovación e inversión, el tercer factor, incluye el número de patentes solicitadas en la OEPM (nacionales), el número de patentes solicitadas en la EPO (europeas) y la inversion en lanzamiento de innovaciones en el mercado. La teoría económica considera las patentes como indicadores de eficiencia a la vez que instrumentos de política industrial destinados a fomentar la innovación y la difusión (68). La evidencia empírica sugiere que las patentes proporcionan una medida bastante fiable de la actividad innovadora (69) ya que la innovación, el crecimiento y la competitividad están correlacionados (69). Por otra parte, en esta nueva variable también se incluyen los gastos relacionados con el lanzamiento de nuevos productos o servicios innovadores. Una vez más, se constata la correlación positiva entre el binomio inversión-innovación. Estudios previos (65) han mostrado que la mejora de las capacidades tecnológicas provoca innovaciones medioambientales. En esta misma línea, Corley et al. (9) encontraron una relación positiva entre la competitividad, la productividad, la inversión y la innovación.

Teniendo en cuenta las nuevas variables obtenidas en base a la agrupación de criterios homogéneos mediante el análisis factorial, se ha aplicado el método de regresión jerárquica (70) para la especificación de un modelo de regresión (véase la tabla III). El tema de la multicolinealidad en el análisis de regresión se resolvió con los análisis factoriales (método Varimax) y, por otra parte, tanto los valores de la tolerancia y la varianza cumplen los requisitos al uso (63).
Los coeficientes de regresión, Beta, son los coeficientes del modelo propuesto y representan la importancia relativa de cada variable en el modelo. Los coeficientes obtenidos son negativos debido a la relación inversa en la base de datos PITEC que considera la importancia de la mejora del impacto ambiental (variable dependiente, ENV-IMP) de forma inversa a su relevancia: muy importante (1), importante (2), no es tan importante (3), no se considera (4) por las empresas a la hora de innovar. Ofrece, por tanto una relacion positiva. La regresión lineal se ajusta a los datos con las nuevas variables obtenidas en el análisis factorial como se desprende del test de significancia (F) del modelo (pie de tabla III).

Así, según este modelo, se puede explicar la probabilidad de la orientación medio ambiental de la empresa en su actividad innovadora según la siguente formula:

Efecto $8=2,426+0,378 *$ Tamaño Empresa $+0,386 *$ Apertura Exterior al Mercado $+0,573$ * Actividad formal Innovación.

Todas las variables tienen un impacto significativo sobre el Impacto Ambiental según el valor obtenido en el test $\mathrm{t}$ de significancia (ver tabla III), aunque es la Actividad formal de innovación e inversión la que presenta una mayor influencia en el efecto final. Así, en base a los datos obtenidos del análisis de regresión lineal, podemos afirmar que nuestra primera hipótesis, H1: Cuanto más orientadas hacia la innovación están las empresas, más orientadas hacia la eco-innovación estarán se cumple, teniendo en cuenta que la actividad innovadora y la inversión formal y es un buen indicador del nivel de innovación y tiene una importancia relevante en la variable dependiente.

Nuestra segunda hipótesis, H2: el tamaño de la empresa determina la orientación hacia la innovación sostenible de la empresa, también se constata. El tamaño aparece como un factor moderador, una característica de las empresas eco-innovadoras, junto con la orientación a la exportación,

TABLA II. RESUMEN DE RESULTADOS DEL ANÁLISIS EXPLORATORIO DE FACTORES.

\begin{tabular}{|c|c|c|c|}
\hline & TAMAÑO & $\begin{array}{l}\text { APERTURA } \\
\text { AL MERCADO }\end{array}$ & $\begin{array}{l}\text { ACTIVIDAD FORMAL DE } \\
\text { INNOVACIÓN E INVERSIÓN }\end{array}$ \\
\hline Ventas netas (NS), & 0,899 & & \\
\hline Inversión en bienes equipo, (INVER) & 0,761 & & \\
\hline Tamaño de la empresa medido por número de empleados, (SZ) & 0,927 & & \\
\hline $\begin{array}{l}\text { Orientación o Enfoque comercial preferente hacia el mercado } \\
\text { nacional, (MDONAC) }\end{array}$ & & 0,713 & \\
\hline $\begin{array}{l}\text { Orientación o Enfoque comercial preferente hacia el mercado } \\
\text { de la Unión Europea, (MDOUE) }\end{array}$ & & 0,794 & \\
\hline Lanzamiento de Innovaciones en el mercado, (MERCADO) & & & 0,642 \\
\hline Número de patentes solicitadas a nivel nacional, (PATOEPM) & & & 0,856 \\
\hline Número de patentes solicitadas a nivel europeo, (PATEPO) & & & 0,820 \\
\hline$\%$ De la varianza explicada & 36,801 & 19,843 & 14,402 \\
\hline
\end{tabular}

* Convergencia en 4 iteraciones ${ }^{* *}$ Análisis de componentes principales. Varimax con normalización Kaiser, 71,046\% de la varianza explicada. Test KMO=0, 671 Nivel de significatividad (- Sig) $p<0,0001$, aceptable si $<0.05$. 
lo que confirma nuestra tercera hipótesis, H3: Cuanto más orientadas a la exportación están las empresas, más sensibles son a los impactos de la eco-innovación.

\section{CONCLUSIONES.}

Esta investigación ha estudiado los factores que influyen en la eco-orientación de los productores de baldosas cerámicas. El estudio empírico se ha realizado en el sector cerámico español, mostrando que el mercado orientado al exterior (no únicamente local), el tamaño de la empresa, la actividad innovadora formal (reflejada por el número de patentes registradas) y la inversión influyen en la orientación ecoinnovadora de las empresas.

Se ha demostrado que las empresas con registros de propiedad industrial (patentes) y mayores inversiones se centran en la innovación, con lo que validamos la primera hipótesis del trabajo. Por otro lado, el análisis de regresión lineal determina que el tamaño de la empresa, medido por el número de empleados, las ventas netas y la inversión total tiene una relación positiva con la orientación sostenible de la empresa, validando también lo propuesto en la segunda hipótesis. Cuanto más amplio es el mercado al que se dirige la empresa, más orientada hacia la eco-innovación está, por lo H3 también se cumple en el estudio realizado.

Los factores que la literatura identifica como impulsores de la innovación, como el tamaño y la apertura al mercado, se puede considerar también como impulsores de la ecoinnovación. De hecho, la innovación, medida tanto a nivel formal a través del número de patentes como por el lanzamiento de nuevos productos de inversión, se revela como el principal factor moderador de la eco-innovación.

Los resultados de esta investigación tienen importantes aplicaciones prácticas por lo que a las actuaciones de política industrial que promueven la eco-innovación se refiere, ya que caracteriza a las empresas susceptibles de aprovechar las actuaciones públicas. La UE, a través de diferentes programas, está financiando proyectos de eco-innovación, principalmente orientados a PYMES. La optimización de estos programas financiados con fondos públicos requiere de una caracterización previa de las empresas que desarrollan procesos de eco-innovación así como identificar los factores que influyen positivamente en una empresa para que pase de ser innovadora a considerar el medioambiente como variable a tener en cuenta y sea eco-innovadora. En este trabajo se ha señalado que sería más eficaz fomentar la eco-innovación en las empresas que ya son innovadoras y que tiene un tamaño significativo.

Este trabajo ha contribuido al cuerpo de conocimiento de la eco-innovación al explicar cómo la estructura interna de la empresa y su tamaño influyen en su orientación hacia la ecoinnovación y cómo la capacidad y la orientación innovadora y la actitud eco-innovadora están relacionadas entre sí, lo que confirma diversas aportaciones previas en la misma dirección $(17,22)$. Por último, ha contribuido al demostrar que la ecoinnovación en la empresa se correlaciona con sus esfuerzos de internacionalización.

Las limitaciones de este estudio se deben, básicamente, a las restricciones de la base de datos. Como actuaciones futuras nos planteamos analizar con mayor profundidad los factores que dificultan o facilitan la eco-innovación a nivel interno y externo de la empresa, así como la influencia de medidas legislativas tales como la Directiva IPPC o la Directiva sobre comercio de emisiones. Por lo tanto, será necesario llevar a cabo estudios de tipo cualitativo más detallados. Por otra parte, sería interesante aplicar el estudio a otros países en los que el sector cerámico tenga una preponderancia similar (por ejemplo Italia) y poder comparar los resultados obtenidos.

Nuestra investigación se ha beneficiado de la utilización de la base de datos PITEC (Panel de Innovación Tecnologica, Ministerio de Industria, Comercio y Turismo) que incluye varios sectores. Sin embargo, debe subrayarse que las conclusiones deben considerarse con prudencia ya que puede haber efectos ocultos debido a si se trata de un sector de baja, media o alta tecnología.

TABLA III. IMPACTO AMBIENTAL DE LA INNOVACIÓN: RESULTADOS DEL ANÁLISIS DE REGRESIÓN.

\begin{tabular}{|c|c|c|c|c|c|c|}
\hline \multirow{2}{*}{\multicolumn{2}{|c|}{ Modelo }} & \multicolumn{2}{|c|}{ Coeficientes no estandarizados } & \multirow{3}{*}{$\begin{array}{r}\begin{array}{r}\text { Coeficient } \\
\text { estandariza }\end{array} \\
\text { Beta }\end{array}$} & \multirow{3}{*}{$\frac{\mathbf{t}}{22,712}$} & \multirow{3}{*}{$\frac{\text { Sig. * }}{0,000}$} \\
\hline & & \multirow{2}{*}{$\frac{\text { B }}{2,426}$} & \multirow{2}{*}{$\begin{array}{c}\text { Std. Error } \\
0,107\end{array}$} & & & \\
\hline & (Constante) & & & & & \\
\hline & TAMAÑO & $-0,378$ & 0,108 & $-0,334$ & $-3,511$ & 0,001 \\
\hline 1 & APERTURA AL MERCADO & $-0,386$ & 108 & $-0,341$ & $-3,581$ & 0,001 \\
\hline & $\begin{array}{l}\text { ACTIVIDAD FORMAL } \\
\text { DE INNOVACIÓN \& INVEF }\end{array}$ & $-0,573$ & 0,108 & $-0,506$ & $-5,323$ & 0,000 \\
\hline
\end{tabular}

a. Variable dependiente: Orientación o enfoque medio ambiental de la empresa, denominada EFECTO8,

${ }^{*}$ Nivel de significatividad Variables Aceptable $\mathrm{si}<0,05$

Nivel de significatividad del modelo $0,000(\mathrm{~F}=17,831)$ Aceptable $\mathrm{si}<0,05$.

Los signos negativos de los coeficientes se deben a la valoración inversa de las respuestas en las variables independientes, así 1 es muy importante y 4 poco importante. 


\section{AGRADECIMIENTOS}

Los autores desean agradecer al Ministerio de Ciencia e Innovación por el apoyo económico recibido a través del proyecto (EC02008-05895-C02-01/ECON) financiado en la convocatoria 2009, así como a la Universidad Politécnica de Valencia por la financiación da través de su programa de financiación de movilidad de profesores y del proyecto PAID06-2011.

\section{REFERENCIAS}

(1) K Rennings, A. Ziegler, K. Ankele, y E. Hoffmann, "The Influence of Different Characteristics of the EU Environmental Management and Auditing Scheme on Technical Environmental Innovations and Economic Performance", Ecological Economics, 57: 45-59, (2006).

(2) J. Bercovitz, y W.Mitchell, "When is more better? The impact of business scale and scope on long-term business survival, while controlling for profitability". Strategic Management Journal.Vol. 28, No 1, pp. 61-79, (2008).

(3) R. Boutellier, O. Gassmann, y M. Zedtwitz, "Managing Global Innovation. Uncovering the Secrets of Future Competitiveness" Springer-Verlag Berlin Heidelberg. Third edition, (2008).

(4) E. Tortajada, D. Gabladón, I. Fernández,"La evolución tecnológica del distrito cerámico de Castellón: la contribución de la industria de fritas, colores y esmaltes", Bol. Soc. Esp. Ceram. V, 47, 2, 57-80 (2008).

(5) G. Van Leeuwen, y L. Klomp, "On the Contribution of Innovation to Multi-Factor Productivity Growth", Economics of Innovation and New Technology, Vol. 15, No 4/5, pp. 367-390 (2006).

(6) F. Crespi y M. Pianta, "Demand and innovation in productivity growth", International Review of Applied Economics.Vol. 22, No. 6, 655-672, (2008).

(7) R. C. Calia, F. M., Guerrini y G. L. Moura, "Innovation networks: From technological development to business model reconfiguration", Technovation, Vol. 27, pp.426-432 (2007)

(8) A. Hidalgo y J. Albors, "Innovation management techniques and tools: a review from theory and practice", R\&D Management; Mar 2008, Vol. 38 Issue 2, p113-127 (2008)

(9) M. Corley, J. Michie y C. Oughton, "Technology, Growth and Employment”, International Review of Applied Economics, Vol. 16, No 3, pp. 265-276 (2002).

(10) J. Baldwin y Z. Lin, "Impediments to advanced technology adoption for Canadian manufacturers", Research Policy 31. Vol 1, pp 1-18 (2002).

(11) P. Mohnen, J. Mairesse y M. Dagenais. "Innovativity: A Comparison Across Seven European Countries." in Empirical Studies of Innovation in the Knowledge Driven Economy, Guest eds. B. Hall and J. Mairesse, Economics of Innovation and New Technologies 15(4-5): 391-413 (2006).

(12) D. Hitchens, S. Thankappan, M. Trainor, J. Clausen, y B. De Marchi, "Environmental performance, competitiveness and management of small businesses in Europe". Royal Dutch Geographical Society, KNAG. Vol. 96, No. 5, pp. 541-557 (2005).

(13) D.C. Esty, y A. S. Winston, "Green to Gold, How smart companies use environmental strategy to innovate, create value, and build competitive advantage", John Wiley and Sons, Hokoben, New Jersey (2006).

(14) M. Beise y K. Rennings, "Lead markets and regulation: a framework for analyzing the international diffusion of environmental innovations", Ecological Economics, Vol. 52, pp. 5-17 (2005).

(15) C. Fussler y P. James, "Driving Eco-Innovation: A Breakthrough Discipline for Innovation and Sustainability", Pitman Publishing: London P. (1996).

(16) M. Jänicke, "Ecological modernisation: new perspectives", Journal of cleaner production. Vol. 16, pp. 557-565 (2008).

(17) OCDE, Sustainable manufacturing and eco-innovation: First steps in building a common analytical framework. DSTI/IND(2008)16/REV1, OECD, Paris (2008).

(18) G. Huppes, "Eco-efficiency: From technical optimisation to reflective sustainability analysis", Ecological Economics, Vol 68, 6, 1687-1700, (2009).

(19) J. González-Benito, "Supply strategy and business performance: An analysis based on the relative importance assigned to generic competitive objectives", International Journal of Operations \& Production Management, Vol. 30 Iss: 8, pp.774 - 797(2010).

(20) J. Vega-Jurado, A. Gutiérrez-Gracia e I. Fernández-de-Lucio, “How do Spanish firms innovate? An empirical evidence. J. Technol. Manag. Innov, Vol. 3, No. 3, pp. 100-111 (2008).

(21) C. H. Anderson, "Hierarchical moderated regression analysis: A useful tool for retail management decisions". Journal of retailing, Vol. 62, No 2, pp.186203 (1986).
(22) R. Kemp y P. Pearson (Eds.), Final report of the project Measuring EcoInnovation (MEI), Available in (http://www.merit.unu.edu/MEI/index. php, (2008).

(23) A. Reid, y M. Miedzinski, “Eco-innovation. Final report for sectoral innovation watch", Technopolis Group .Bruselas (2008).

(24) J. Molero, A.Garcia, The innovative activity of foreign subsidiaries in the Spanish Innovation System: An evaluation of their impact from a sectoral taxonomy approach. Technovation 28, 739-757 (2008).

(25) P. Soto-Acosta, A. Luis Meron-Cerdan, "Analyzing e-business value creation from a resource-based perspective", International Journal of Information Management, 28, 49-60 (2008).

(26) H.C Wang, J. He, J. y J. T. Mahoney, "Firm-specific knowledge resources and competitive advantage: the roles of economic- and relationship-based employee governance mechanisms", Strategic Management Journal. Vol. 30, No 12, pp. 1265-1285 (2009).

(27) G. Noci y R. Verganti, “Managing 'green' product innovation in small firms", R\&D Management; Vol. 29,N 1, pp. 3-15 (1999).

(28) C. McKeiver, D. Gadenne, "Environmental management systems in small and medium businesses", International Small Business Journal, Vol 23, n 5 , 513-537 (2005).

(29) I. Martín-Tapia, J. A. Aragón-Correa y A. Rueda-Manzanares, "Environmental strategy and exports in medium, small and microenterprises", Journal of World Business, Vol. 45, N3, 266-275 (2010).

(30) M. Abbaspour, A. R. Karbassi y S. Khadivi, "Implementation of green management concepts in sport complexes", Int. J. Environ. Sci. Tech., Vol 3, No 3, pp. 213-219 (2006).

(31) R. Beveridge y S. Guy, "The rise of the eco-preneur and the messy world of environmental innovation", Local Environmetal, Vol. 10, No 6, pp. 665-676 (2005).

(32) S. Hallstedt, H. Ny, K. H. Robèrt y G. Broman, “An approach to assessing sustainability integration in strategic decision systems for product development", Journal of Cleaner Production, Vol. 18, No 8, 703-712 (2010).

(33) M. Kanerva, A. Arundel y R. Kemp, "Environmental innovation: Using qualitative models to identify indicator for policy", United Nations University, Working Papers Series (2009).

(34) A. Arundel y R. Kemp, "Measuring Eco-innovation", Working paper series. United Nations University, Maastricht (2009).

(35) D. Pujari, "Eco-innovation and new product development: understanding the influences on market performance", Technovation, Vol. 26, pp. 76-85 (2006).

(36) F. Steward, "Direct measurement of innovation output using documentary and digital sources". Report for measuring eco-innovation project. European Commission and European Environment Agency, Bruselas (2008).

(37) N. Schwarz, A. Ernst, "Agent-based modelling of the difussion of environmental innovations-An empirical approach", Technological Forecasting and Social Change, Vol. 76, pp.497-511 (2009).

(38) A. Jaffe, K. Palmer, "Environmental Regulation and Innovation: A Panel Data Study", Review of Economics and Statistics, Vol. 79, No. 4, 610-619 (1997).

(39) M. Wagner, "Sustainability-related innovation and sustainability management. A quantitative analysis", Centre for Sustainability Management. Lüneburg (2008).

(40) M. Segarra-Oña, A. Peiró-Signes, J. Albors-Garrigós y L. Miret-Pastor, "Impact of innovative practices in environmental focused firms. Moderating factors", International Journal of Environmental Research, Vol 5 No 2, 425-434 (2011).

(41) J. E. Ettlie, W. P. Bridges, y R. D. O’Keefe, “Organization strategy and structural differences for radical versus incremental innovation",Management Science, vol. 30, No 6 682-695(42) J. E Ettlie y A. H. Rubenstein, "Firm size and product innovation", Journal of Product Innovation Management., Vol. 4, No 2, pp. 89-108 (1987).

(43) N. Lazaric, y B. Dennis, B. 'Routinisation and memorisation of tasks inside a workshop: an illustration through a case study', Industrial and Corporate Change, Vol. 14, No. 5, 873-896 (2005).

(44) R. R. Nelson y S. G. Winter, "Evolutionary Theorizing in Economics", Journal of Economic Perspectives, Vol. 16, No 2, 23-46 (1982).

(45) N. C. Churchill y V. L. Lewis, "The five stages of small business growth", Harvard Business Review; May/Jun83, Vol. 61, N 3, 30-50 (1983).

(46) A. Reid y K. Männik, "How do sectorial patterns influence companies innovation management practice in Europe" Technopolis group.Bruselas (2008)

(47) V. Biondi, F. Iraldo, S. Meredith, "Achieving sustainability through environmental innovation: the role of SMEs", Int. J. Technology Management, Vol. 24, No 5/6, 612-626 (2002).

(48) J. A. Brio y B. A. Junquera, "Review of the literature on environmental innovation management in SMEs: Implications for public policies", Technovation, 23: 939-948 (2003).

(49) S. B. Brunnermeier y M. A. Cohen, "Determinants of environmental innovation in US manufacturing industries", Journal of Environmental Economics and Management. Vol. 45, 278-293 (2003). 
(50) K. Rennings, "Redefining innovation - eco-innovation research and the contribution from ecological economics", Ecological Economics, Vol 32, 2 319-332 (2002).

(51) M. Jänicke, "Trend-setters in environmental policy: the character and role of pioneer countries", European Environment, Vol. 15, No 2, pp. 129-142 (2005).

(52) T. Hellström, T., "Dimensions of environmentally sustainable innovation: the structure of eco-innovation concepts", Sustainable Development, Vol15,N 3, 148-159 (2007).

(53) L. E. Greiner, "Evolution and Revolution as Organizations Grow: A company's past has clues for management that are critical to future success", Family Business Review, vol. 10, no.4, Reprinted by permission of Harvard Business Review. From "Evolution and Revolution as Organizations Grow" by Larry E. Greiner, July-August 1972, (1997).

(54) ASCER, "Información económica del sector español de baldosas cerámicas", Información económica. En http: / / www.ceramicadeespaña. es (2010).

(55) J. Albors-Garrigós, J. L. Hervas-Oliver, "La industria cerámica europea en el siglo XXI. Retos tecnológicos y desafíos de la próxima década" Bol. Soc. Esp. Ceram. V. 45, 1, 13-21 (2006).

(56) E Criado, "Reflexiones sobre el futuro de la Industria Europea de la Cerámica", Bol. Soc. Esp. Ceram. V., 46, 1, 39-44 (2007).

(57) E. Vallada, C. Maroto, R. Ruiz y B. Segura, “Análisis de la programación de la producción en el sector cerámico español”, Bol. Soc. Esp. Ceram. V., 44, 1, 39-44 (2005).

(58) B. Segura, E. Vallada, C. Maroto y R. Ruiz, "Análisis del sistema de operaciones en empresas del sector cerámico español", Bol. Soc. Esp. Ceram.V., 45,1, 13-21 (2006)

(59) J. Albors, J. L. Hervás y P. Márquez, “Adopción contingente de tecnología de producción en el sector cerámico español. Un estudio empírico", Bol. Soc.Esp. Ceram. V., 45, 5, 338-345 (2006).
(60) J. Albors-Garrigós, P. Marquez-Rodríguez y J. L. Hervas-Oliver, "Las relaciones fabricante distribuidor como elementos básicos de un modelo competitivo en el caso del cluster cerámico español. Análisis empírico de los factores moderadores", Bol. Soc. Esp. Ceram. V., 47, 6, 339-344. (2008).

(61) J. Albors-Garrigós, P. Márquez-Rodríguez, M. Segarra-Oña, "Internet como herramienta de creación de valor en sectores maduros. El caso de los productores y distribuidores cerámicos en España", Bol. Soc. Esp. Ceram. V. 48, 6, 273-278 (2009)

(62) L. Dubé y G. Paré, "Rigor in IS Positivist Case Research: Current Practices, Trends and Recommendations, MIS Quarterly Vol. 27, No. 4 pp. 597-635 (2003).

(63) J.F. Hair, R.E. Anderson, R.L. Tatham y W.C. Black, "Multivariate Date Analysis", 5th ed., Prentice-Hall, Englewood Cliffs, NJ. (1998).

(64) M. C Becker, N. Lazaric, R. R. Nelson, y S. G. Winter, "Applying organizational routines in understanding organizational change", Industrial and Corporate Change. Vol. 14, pp. 1-17 (2005).

(65) J. Horbach, "Determinants of Environmental Innovation.New Evidence from German Panel Data Sources", Research Policy 37, 163-173 (2008).

(66) R. Bassile, "Export behaviour of Italian manufacturing firms over the nineties: the role of innovation", Research Policy, Vol 30, 8 pp 1185-1201 (2001).

(67) R. Salomon y M. Shaver, "Learning by exporting: new insights from examining firm innovation. Journal of Economics and Managemen Strategy. Vol 14, 2, 693-713 (2005)

(68) D. Encaoua, D. Guellec y C. Martínez, "Patent systems for encouraging innovation: Lessons from economic analysis", Research Policy. Vol. 35, No 9, pp. 1423-1440 (2006)

(69) M. Crosby, "Patents, Innovation and Growth", Economic Record. Vol. 76, No. 234, pp. 255-262 (2007).

(70) J. Cohen y P. Cohen, “Applied Multiple Regression/Correlation. Analysis for the Behavioral Sciences, second ed. Lawrence Erlbaum, New Jersey (1983).

Recibido: $21 / 12 / 2010$

Aceptado: 08/09/2011 